\title{
Eficiência de fontes e doses de fósforo na alfafa e centrosema cultivadas em Latossolo Amarelo(1)
}

\begin{abstract}
Adônis Moreira ${ }^{(2)}$, Eurípedes Malavolta ${ }^{(3)}$ e Larissa Alexandra Cardoso Moraes(2)
Resumo - O objetivo deste trabalho foi avaliar a eficiência agronômica de fontes e doses de P na alfafa e na centrosema, cultivadas em casa de vegetação, em Latossolo Amarelo distrófico. O delineamento experimental foi inteiramente casualizado em esquema fatorial $4 \mathrm{x} 4$ : quatro fontes de $\mathrm{P}$ (superfosfato triplo, termofosfato Yoorin, fosfato natural da Carolina do Norte e fosfato natural de Arad) e quatro doses de $\mathrm{P}\left(0,50,100\right.$ e $\left.200 \mathrm{mg} \mathrm{kg}^{-1}\right)$, com três repetições, sendo realizados seis cortes. A eficiência das fontes e doses de P foi determinada por três métodos: Índice de Eficiência Agronômica, Equivalente Superfosfato Triplo e Índice de Eficiência; o P recuperado foi avaliado por Mehlich 1, Mehlich $3 \mathrm{e}$ resina. Os melhores índices de Eficiência Agronômica e Equivalente Superfosfato Triplo foram obtidos com o termofosfato Yoorin. O Índice de Eficiência foi maior na centrosema do que na alfafa. Com a sequiência de cortes, a produção de matéria seca obtida com os fosfatos naturais reativos tende a equiparar-se à das fontes mais solúveis. O termofosfato Yoorin eleva o $\mathrm{pH}$ do solo, enquanto o superfosfato triplo o diminui; os fosfatos naturais apresentam reação neutra.
\end{abstract}

Termos para indexação: Centrosema pubescens, Medicago sativa, adubo fosfatado, extrator, acidificação.

\section{Efficiency of phosphorus sources and rates for alfalfa and centrosema cultivated in an Yellow Latosol (Oxisol)}

\begin{abstract}
A greenhouse experiment was carried out to evaluate the efficiency of phosphorus sources and rates for alfalfa and centrosema in an dystrophic Yellow Latosol (Oxisol). A completely randomized design in a $4 \times 4$ factorial was used: four sources of $\mathrm{P}$ (triple superphosphate, Yoorin magnesium thermophosphate, Arad rock phosphate, and North Caroline rock phosphate) and four rates of $\mathrm{P}(0$, 50,100 and $200 \mathrm{mg} \mathrm{kg}^{-1}$ ) with three replicates. Six harvests were made. The efficiency of the phosphatic fertilizers was evaluated by the methods: Index of Agronomic Efficiency, Triple Superphosphate Equivalent, and Efficiency Index. Recovered P was evaluated by the Mehlich 1, Mehlich 3 and ion-exchange resin methods. The best Index of Agronomic Efficiency and Triple Superphosphate Equivalent were obtained for Yoorin magnesium thermophosphate. The Efficiency Index values were higher for centrosema than for alfalfa. When sequence harvest is used, the dry matter production obtained with the natural reactive phosphates tends to match the production obtained with more soluble sources. Yoorin magnesium thermophosphate increased, whereas triple superphosphate decreased soil $\mathrm{pH}$; the rock phosphate had neutral reaction.
\end{abstract}

Index terms: Centrosema pubescens, Medicago sativa, phosphate fertilizers, extractor, acidification.

(1) Aceito para publicação em 10 de junho de 2002 .

Extraído da dissertação de mestrado apresentada pelo primeiro autor à Escola Superior de Agricultura Luiz de Queiroz, Piracicaba, SP. Parcialmente financiado pela Fapesp.

(2) Embrapa-Centro de Pesquisa Agroflorestal da Amazônia Ocidental, Caixa Postal 319, CEP 69011-970 Manaus, AM. E-mail: adonis@cpaa.embrapa.br, larissa@cpaa.embrapa.br

(3) Universidade de São Paulo, Centro de Energia Nuclear na Agricultura, Caixa Postal 96, CEP 13400-970 Piracicaba, SP. Bolsista do CNPq. E-mail: mala@cena.usp.br

\section{Introdução}

Espécies forrageiras podem diferir na capacidade de absorção de determinado elemento, tanto pela desigualdade na constante que define a afinidade entre o elemento e o seu carregador como pela distribuição do sistema radicular, ou de ambas (Fenster \& León, 1982). Lobato et al. (1986) observaram que os níveis críticos de $\mathrm{P}$ no solo para as forrageiras tropicais são mais baixos do que para as leguminosas de clima temperado. As primeiras espécies são nativas 
de regiões com solos pobres em $\mathrm{P}$ disponível, enquanto as segundas são originárias de regiões com solos apresentando alto teor de P, além de outros nutrientes. Na escolha de forrageiras, é essencial que se considere, além desses fatores, o clima, as condições de solo de cada região e, principalmente, a fonte de $\mathrm{P}$ a ser utilizada, tendo em vista o papel desse nutriente no estabelecimento, enraizamento e brotação das forrageiras (Guss et al., 1990).

Os adubos fosfatados mais utilizados atualmente na agricultura brasileira são os fosfatos solúveis, termofosfatos, multifosfatos e fosfatos naturais. No Brasil também têm sido fabricados fertilizantes fosfatados parcialmente acidulados. Produtos de origem orgânica têm sido complementos de grande valor (Malavolta, 1980). Por isso, a escolha de uma fonte, normalmente, se baseia tanto na sua eficiência em suprir P para as plantas como na sua relação custo:benefício (Goedert et al., 1985).

Segundo Coelho \& Verlengia (1973), os fosfatos naturais são fertilizantes que apresentam baixa disponibilidade de $\mathrm{P}$ para as plantas no início do cultivo, restringindo a sua utilização na agricultura. As principais fontes de fosfatos naturais são as apatitas e fosforitas. As primeiras são originárias de minerais primários e de baixo teor de $\mathrm{P}$ disponível (cerca de 3\%), enquanto as fosforitas são minerais fosfatados amorfos de origem secundária; sua solubilidade em citrato de amônio está em torno de 5\%.

Os fertilizantes solúveis em água são obtidos pela acidulação de rochas fosfatadas, e destacam-se o superfosfato simples que contém cerca de $9 \%$ de P, e o superfosfato triplo com aproximadamente $20 \%$ de fósforo. Os dois representam, aproximadamente, $50 \%$ dos fertilizantes fosfatados fabricados no mundo (Fassbender \& Bornemisza, 1994). Já o termofosfato possui cerca de $8 \%$ de $\mathrm{P}$, sendo a sua solubilidade grandemente influenciada pelo $\mathrm{pH}$ e pela matéria orgânica do solo. Por ser uma fonte rica em Ca e em $\mathrm{Mg}$, atua muitas vezes como corretivo da acidez do solo (Goedert et al., 1985).

$\mathrm{O}$ adubo fosfatado adicionado ao solo, além do efeito imediato sobre a cultura que se segue à adubação, pode ter um efeito residual nos cultivos subseqüentes. Além do tipo de cultura, vários fatores podem afetar o efeito residual dos adubos fosfatados, tais como: doses e fontes de P, método de aplicação, manejo, temperatura, tipo de solo, tempo de aplicação e umidade do solo. Ao contrário dos fosfatos solúveis e dos termofosfatos, os fosfatos naturais apresentam uma solubilização mais lenta, podendo ocorrer um aumento gradativo da disponibilidade de P (Novais \& Smyth, 1999).

O objetivo deste trabalho foi avaliar a eficiência agronômica de fontes e doses de $\mathrm{P}$ na alfafa e na centrosema em Latossolo Amarelo distrófico, cultivados em casa de vegetação.

\section{Material e Métodos}

Os experimentos foram realizados no período de dezembro de 1995 a agosto de 1996, em casa de vegetação do Centro de Energia Nuclear na Agricultura (Cena/USP), no Município de Piracicaba, Estado de São Paulo.

Utilizou-se o delineamento experimental inteiramente casualizado em esquema fatorial $4 \times 4$, sendo quatro fontes de P: superfosfato triplo, SPT; termofosfato Yoorin, TY; fosfato natural da Carolina do Norte, FNCN e fosfato natural de Arad, FNA (Tabela 1) e quatro doses de P (0, 50,100 e $200 \mathrm{mg} \mathrm{kg}^{-1}$ ), em seis épocas de cortes com intervalos de 30 dias, com três repetições. O solo utilizado foi um Latossolo Amarelo distrófico, coletado $(0-20 \mathrm{~cm})$ no Município de Nova Odessa, Estado de São Paulo, peneirado em peneira de malha de $4,0 \mathrm{~mm}$ com as seguintes características físico-químicas: $\mathrm{pH} \mathrm{em} \mathrm{CaCl} 2\left(0,01 \mathrm{~mol} \mathrm{~L}^{-1}\right), 3,7$; MO, 20,3 $\mathrm{g} \mathrm{kg}^{-1}$; $\mathrm{P}_{\text {(resina) }}, 4,0 \mathrm{mg} \mathrm{dm}^{-3}$; $\mathrm{P}_{\text {(Mehlich 1) }}$, $6,0 \mathrm{mg} \mathrm{dm}^{-3} ; \mathrm{P}_{\text {(Mellich } 3)}, 5,0 \mathrm{mg} \mathrm{dm}^{-3} ; \mathrm{S}_{-} \mathrm{SO}_{4}{ }^{2-}, 59 \mathrm{mg} \mathrm{kg}^{-1}$; $\mathrm{K}^{+}, 1,0 \mathrm{mmol}_{\mathrm{c}} \mathrm{dm}^{-3} ; \mathrm{Ca}^{2+}, 3,0 \mathrm{mmol}_{\mathrm{c}} \mathrm{dm}^{-3} ; \mathrm{Mg}^{2+}$, $2,0 \mathrm{mmol}_{\mathrm{c}} \mathrm{dm}^{-3} ; \mathrm{Al}^{3+}, 21 \mathrm{mmol}_{\mathrm{c}} \mathrm{dm}^{-3} ; \mathrm{H}^{+}+\mathrm{Al}^{3+}$, $96 \mathrm{mmol}_{\mathrm{c}} \mathrm{dm}^{-3}$; V\%, 6,0; P orgânico, $3,9 \mathrm{mg} \mathrm{dm}^{-3}$; capacidade máxima de adsorção, $715,1 \mathrm{mg} \mathrm{kg}^{-1} \mathrm{e}$ densidade, $1,2 \mathrm{~g} \mathrm{~cm}^{-3}$ (Embrapa, 1997).

Foram utilizados vasos de barro com cinco quilos de capacidade, impermeabilizados internamente com neutrol e providos de coletores para impedir a lixiviação dos nutrientes. Cada leguminosa foi mantida em bancada distinta. A acidez do solo foi corrigida 30 dias antes do plantio, visando elevar o índice de saturação por bases ao valor de $70 \%$ para alfafa e $50 \%$ para centrosema, empregando-se calcário dolomítico com $400 \mathrm{~g} \mathrm{~kg}^{-1}$ de $\mathrm{CaO}, 150 \mathrm{~g} \mathrm{~kg}^{-1}$ de $\mathrm{MgO}, \mathrm{PN} 108 \%$ e PRNT $86 \%$. Exceto o P e o N, a adubação com os demais nutrientes foi a recomendada por Malavolta (1980), para experimentos realizados em casa de vegetação: $\mathrm{K}, 50 \mathrm{mg} \mathrm{kg}^{-1}$;, $50 \mathrm{mg} \mathrm{kg}^{-1}$; $, 0,5 \mathrm{mg} \mathrm{kg}^{-1}$; $\mathrm{Cu}, 1,5 \mathrm{mg} \mathrm{kg}^{-1}$; Fe, 5,0 $\mathrm{mg} \mathrm{kg}^{-1}$; Mn, 5,0 $\mathrm{mg} \mathrm{kg}^{-1}$ e Zn, $5,0 \mathrm{mg} \mathrm{kg}^{-1}$. Após o segundo e o quarto corte, aplicaramse $50 \mathrm{mg} \mathrm{kg}^{-1}$ de $\mathrm{K}_{2} \mathrm{SO}_{4}$ e no terceiro corte, os 
micronutrientes (B, Cu, Mn e $\mathrm{Zn}$ ) na mesma dose da adubação de plantio.

Após a escarificação das sementes da alfafa e da centrosema, estas foram submetidas à inoculação de Rhizobium meliloti e Rhizobium centrosema, respectivamente, e tratadas com $0,1 \mathrm{mg} \mathrm{L}^{-1}$ de Co e $0,1 \mathrm{mg} \mathrm{L}^{-1}$ de Mo. Em cada vaso, foram mantidas cinco plantas uniformes. Após três meses do plantio, foram realizados na alfafa e na centrosema, na altura de $10 \mathrm{~cm}$ do solo, seis cortes consecutivos com intervalo de 30 dias. As raízes foram coletadas após o sexto corte.

Na determinação da quantidade de $\mathrm{P}$ disponível, amostras de solo de cada tratamento foram coletadas em cada época de corte e analisadas, empregando-se três extratores: método da resina trocadora de íons (Raij et al., 1986), Mehlich 1 (Mehlich, 1978) e Mehlich 3 (Mehlich, 1984). Nas mesmas amostras, determinou-se o pH em solução 0,01 mol L-1 de $\mathrm{CaCl}_{2}$ (Embrapa, 1997).

A avaliação das respostas das culturas aos adubos fosfatados foi realizada mediante cálculo do Índice de Eficiência Agronômica (IEA) e Equivalente Superfosfato Triplo (EqSFT), descritos por Chien \& Hammond (1978) e Goedert et al. (1985):

$\operatorname{IEA}(\%)=100\left(\mathrm{Pn}-\mathrm{P}_{0}\right) /\left(\mathrm{P}_{\mathrm{SFT}}-\mathrm{P}_{0}\right)$ e

$\operatorname{EqSFT}(\%)=100 \mathrm{P}_{\mathrm{SFT}} / \mathrm{Pn}$,

sendo: $\mathrm{P}_{\mathrm{n}}$, a produção obtida com a fonte na dose $\mathrm{n} ; \mathrm{P}_{0}$, a produção da testemunha $\left(0 \mathrm{mg} \mathrm{kg}^{-1} \mathrm{de} \mathrm{P}\right)$; $\mathrm{P}_{\mathrm{SFT}}$, a produção obtida com a fonte solúvel na dose $n$.

Além desses, foi determinado na soma dos seis cortes, o Índice de Eficiência (IE). A produção foi ajustada a um polinômio do $2^{\mathrm{o}}$ grau com as doses de $\mathrm{P}$ como variável independente, sendo a derivada $\mathrm{dy} / \mathrm{dx}$ utilizada para determinar a produção máxima estimada e a dose estimada para a máxima produção com os seguintes procedimentos:

$$
\begin{aligned}
& \frac{\mathrm{dy}}{\mathrm{dx}}=\mathrm{ax}^{2}+\mathrm{bx}+\mathrm{c} ; \\
& \mathrm{IE}=\frac{\text { Produção máxima estimada }}{\text { Dose estimada para a máxima produção }},
\end{aligned}
$$

sendo: $\mathrm{x}$, a dose estimada para a máxima produção em $\mathrm{mg} \mathrm{kg}^{-1}$; e y, a produção máxima estimada em gramas por 5,0 kg de solo (quantidade de solo no vaso).

A taxa de recuperação do $\mathrm{P}$ aplicado foi determinada na última amostragem conforme Anghinoni \& Bohen (1974):

$$
\% \text { de } \mathrm{P} \text { recuperado }=\frac{\mathrm{P} \text { na análise }-\mathrm{P} \text { nativo }}{\mathrm{P} \text { adicionado }} \times 100 \text {. }
$$

Os resultados foram submetidos à análise de variância (teste F), ao teste de comparação de contraste entre médias (Tukey 5\%) e regressão, conforme Pimentel-Gomes (1990).

\section{Resultados e Discussão}

Em alfafa, o termofosfato Yoorin (TY) apresentou o mais alto IEA na soma dos seis cortes (Tabela 2), o que corrobora os resultados obtidos em outras culturas por Goedert \& Lobato (1984) e Goedert et al. (1990). Conforme Alcarde \& Ponchio (1979), a solubilidade do termofosfato em ácido cítrico e em citrato neutro de amônio é elevada; quanto maior for a solubilidade em ácidos orgânicos, mais rápida deve ser a difusão do $\mathrm{P}$ no processo de absorção pelas plantas, como também a adsorção pelas partículas do solo.

Os fosfatos naturais da Carolina do Norte (FNCN) e de Arad (FNA) na dose $100 \mathrm{mg} \mathrm{kg}^{-1}$ de $\mathrm{P}$ apresentaram IEA superiores ao do superfosfato triplo na alfafa (Tabela 2). Nas doses 50 e $200 \mathrm{mg} \mathrm{kg}^{-1}$ de P, o mesmo não ocorreu, sendo estes inferiores ao SFT e ao TY. Com a seqüência dos cortes, os fosfatos naturais aumentaram a eficiência, na média das doses, em $38 \%$, enquanto o termofosfato decresceu $36 \%$, quando comparado com o superfosfato triplo.

Na centrosema, na dose $50 \mathrm{mg} \mathrm{kg}^{-1} \mathrm{de} \mathrm{P}$, o FNCN,

\begin{tabular}{|c|c|c|c|c|}
\hline Característica & FNCN & FNA & TY & SFT \\
\hline & & - & & 4300 \\
\hline $\begin{array}{l}\mathrm{P}_{2} \mathrm{O}_{5} \text { total } \\
\mathrm{P}_{2} \mathrm{O}_{5} \text { solúvel em água }\end{array}$ & $\begin{array}{r}300,0 \\
0,0\end{array}$ & $\begin{array}{r}330,0 \\
0,0\end{array}$ & $\begin{array}{r}180,0 \\
0,0\end{array}$ & $\begin{array}{l}430,0 \\
379,0\end{array}$ \\
\hline $\mathrm{P}_{2} \mathrm{O}_{5}$ solúvel em ácido cítrico & 93,0 & 99,6 & 165,0 & 390,0 \\
\hline $\mathrm{P}_{2} \mathrm{O}_{5}$ solúvel em citrato + água & 53,3 & 29,1 & 75,0 & 416,0 \\
\hline Ca total & 328,5 & 371,4 & 192,5 & 221,8 \\
\hline Mg total & 0,0 & 12,0 & 86,6 & 0,0 \\
\hline
\end{tabular}
o FNA e o TY apresentaram IEA superior ao do SFT; no EqSFT, somente o FNA foi estatisticamente dife-

Tabela 1. Características dos adubos fosfatados em diferentes fontes ${ }^{(1)}$.

(1)FNCN: fosfato natural da Carolina do Norte; FNA: fosfato natural de Arad; TY: termofosfato Yoorin; SFT: superfosfato triplo. 


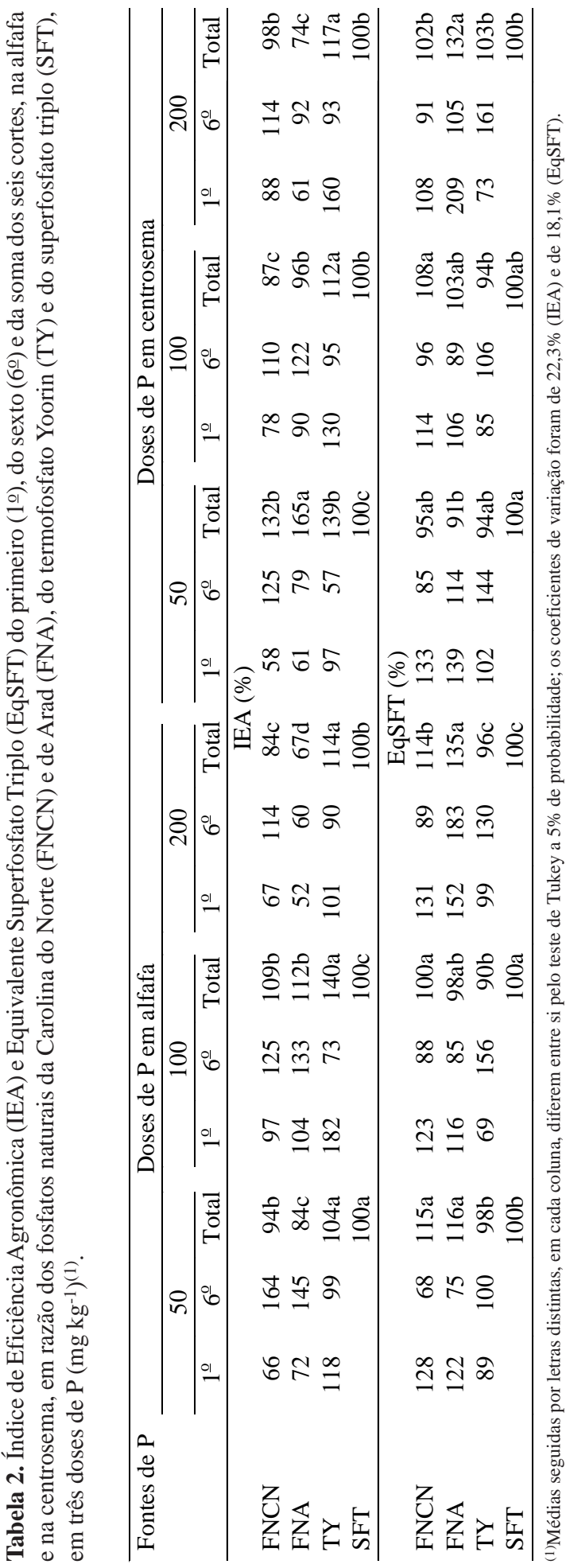

rente do SFT, o que não ocorreu nas doses de 100 e $200 \mathrm{mg} \mathrm{kg}^{-1}$ de fósforo. Nesta leguminosa, os fosfatos naturais aumentaram a eficiência do primeiro ao sexto corte quando comparado com o superfosfato triplo, na média das doses, em $32 \%$, enquanto o termofosfato decresceu $37 \%$ (Tabela 2). Segundo Tisdale et al. (1993), dependendo do tipo de solo, tipo de cultura e manejo, os fosfatos naturais, com alta, média e baixa solubilidade em citrato, apresentam faixas de eficiência entre $80 \%$ a $100 \%$, $50 \%$ a $80 \%$ e $30 \%$ a $50 \%$, respectivamente, comparativamente à resposta inicial da cultura ao superfosfato triplo, aumentando a eficiência com o passar do tempo.

Os IEA e EqSFT obtidos na alfafa e na centrosema concordam com os de Chien \& Hammond (1978), indicando, assim, que o efeito residual dos fosfatos naturais reativos tende a equiparar-se ao das fontes fosfatadas mais solúveis, o que pode ser atribuído ao esgotamento natural destas fontes, além da acidificação do solo favorecendo a solubilização dos fosfatos naturais.

A centrosema apresentou maior Índice de Eficiência, necessitando, com exceção do FNA, de doses menores para obtenção do máximo de produção de biomassa seca (Tabela 3). O aproveitamento do P por esta leguminosa foi, na média, cerca de $43 \%$ maior que pela alfafa. Guss et al. (1990), em condições de casa de vegetação com cinco tipos de solos, obtiveram melhor eficiência na utilização do $\mathrm{P}$ pela centrosema quando comparada com outras leguminosas testadas. Segundo Föhse et al. (1991), a diferença entre as espécies na absorção de $\mathrm{P}$ pode ser atribuída, principalmente, à morfologia do sistema radicular. No caso da centrosema, a maior eficiência na utilização do $\mathrm{P}$ (5,3 mg de $\mathrm{P}$ na planta/g de raiz) decorreu, principalmente, da menor produção de raízes $(5,5 \mathrm{~g})$ quando comparada com a alfafa $(8,8 \mathrm{~g})$, o que resultou numa menor eficiência na absorção de P (3,7 mg de P na planta/g de raiz).

$\mathrm{Na}$ centrosema, os extratores ácidos apresentaram maior taxa de recuperação do $\mathrm{P}$ (Mehlich 1 nos fosfatos naturais e Mehlich 3 no termofosfato Yoorin e no superfosfato triplo) do que a resina, sendo superiores no FNCN e no SFT (Tabela 4). Segundo Shelton \& Coleman (1968), os métodos que utilizam fluoretos na sua composição (Mehlich 3) são indicados para determinação do fósforo recuperado e para 
extração de fosfatos ligados a $\mathrm{Fe}$ e $\mathrm{Al}$, enquanto o Mehlich 1 (Mehlich, 1978) apresenta ação preferencial na dissolução de fosfatos de cálcio. Muzzili (1982), trabalhando com fosfatos naturais, encontrou altos teores de P extraído pelo Mehlich 1, sem que ocorresse elevada eficiência do uso deste nutriente pelas culturas. Com relação à resina, esse extrator, a princípio, retira o $\mathrm{P}$ apenas nas formas solúveis e lábeis, não superestimando a quantidade de $\mathrm{P}$ disponível para as plantas (Raij, 1978).

No caso da alfafa, o extrator resina apresentou maior capacidade de recuperação do $\mathrm{P}$ adicionado

Tabela 3. Produção de matéria seca (MS) da parte aérea $\left(\mathrm{g} / \mathrm{vaso}^{-1}\right)$, estimada pela equação de regressão da soma de seis colheitas, e Índice de Eficiência da alfafa e da centrosema, em relação às diferentes fontes e doses de fósforo.

\begin{tabular}{lccc}
\hline Fontes de P & $\begin{array}{c}\text { Dose de } \mathrm{P}^{(1)} \\
\left(\mathrm{mg} \mathrm{kg}^{-1}\right)\end{array}$ & Produção de MS & Índice de Eficiência \\
\hline Fosfato natural da Carolina do Norte & 172,0 & Alfafa & 48,6 \\
Fosfato natural de Arad & 107,8 & 41,8 & 63,1 \\
Termofosfato Yoorin & 170,8 & 34,0 & 58,4 \\
Superfosfato triplo & 197,5 & 49,9 & 44,7 \\
\hline & & 44,1 & 84,3 \\
Fosfato natural da Carolina do Norte & 158,3 & Centrosema & 97,5 \\
Fosfato natural de Arad & 137,8 & 66,7 & 99,2 \\
Termofosfato Yoorin & 152,5 & 67,2 & 95,2 \\
Superfosfato triplo & 136,0 & 75,6 & 64,7 \\
\hline
\end{tabular}

${ }^{(1)}$ Dose de P para obtenção da máxima produção de matéria seca.

Tabela 4. Porcentagem média de $\mathrm{P}$ recuperado, de diferentes fontes de $\mathrm{P}$, pelos extratores Mehlich 1, Mehlich 3 e resina, após o sexto corte da alfafa e da centrosema ${ }^{(1)}$.

\begin{tabular}{|c|c|c|c|c|c|c|}
\hline \multirow{2}{*}{$\begin{array}{l}\text { Fósforo } \\
\left(\mathrm{mg} \mathrm{kg}^{-1}\right)\end{array}$} & \multicolumn{3}{|c|}{ Alfafa } & \multicolumn{3}{|c|}{ Centrosema } \\
\hline & Mehlich 1 & Mehlich 3 & Resina & Mehlich 1 & Mehlich 3 & Resina \\
\hline & \multicolumn{6}{|c|}{ Fosfato natural da Carolina do Norte } \\
\hline 50 & 14,0 & 12,0 & 18,0 & 12,0 & 8,0 & $-2,0$ \\
\hline 100 & 30,0 & 11,0 & 30,0 & 33,0 & 19,0 & 18,0 \\
\hline 200 & 42,0 & 14,0 & 38,5 & 36,0 & 23,5 & 20,5 \\
\hline Média & $28,7 \mathrm{a}$ & $12,3 \mathrm{~b}$ & $28,8 \mathrm{a}$ & $27,0 \mathrm{~A}$ & $16,8 \mathrm{~B}$ & $12,2 \mathrm{~B}$ \\
\hline & \multicolumn{6}{|c|}{ Fosfato natural de Arad } \\
\hline 50 & 16,0 & 8,0 & 40,0 & 16,0 & 12,0 & 12,0 \\
\hline 100 & 21,5 & 14,0 & 41,0 & 31,0 & 15,0 & 23,0 \\
\hline 200 & 21,5 & 13,0 & 41,0 & 39,0 & 18,0 & 36,5 \\
\hline Média & $19,7 \mathrm{~b}$ & $11,7 \mathrm{c}$ & $41,5 \mathrm{a}$ & $28,7 \mathrm{~A}$ & $15,0 \mathrm{~B}$ & $23,8 \mathrm{~A}$ \\
\hline & \multicolumn{6}{|c|}{ Termofosfato Yoorin } \\
\hline 50 & 8,0 & 6,0 & 10,0 & 2,0 & 4,0 & 2,0 \\
\hline 100 & 11,0 & 11,0 & 17,0 & 11,0 & 11,0 & 15,0 \\
\hline 200 & 28,5 & 15,5 & 23,0 & 24,0 & 29,0 & 20,5 \\
\hline Média & $15,8 \mathrm{a}$ & $10,8 \mathrm{~b}$ & $16,7 \mathrm{a}$ & $12,3 \mathrm{~A}$ & $14,7 \mathrm{~A}$ & $12,5 \mathrm{~A}$ \\
\hline & \multicolumn{6}{|c|}{ Superfosfato triplo } \\
\hline 50 & 12,0 & 12,0 & 28,0 & 10,0 & 10,0 & $-2,0$ \\
\hline 100 & 14,0 & 17,0 & 24,0 & 14,0 & 22,0 & 18,0 \\
\hline 200 & 31,5 & 42,0 & 37,5 & 27,0 & 36,0 & 27,0 \\
\hline Média & $19,2 b$ & $23,7 \mathrm{ab}$ & $29,8 \mathrm{a}$ & $17,0 \mathrm{AB}$ & $22,7 \mathrm{~A}$ & $14,3 \mathrm{~B}$ \\
\hline
\end{tabular}


do que os extratores Mehlich 1 e Mehlich 3 (Tabela 4). Assim como ocorreu com o IEA, a elevação da saturação por bases a 70\%, na alfafa, comparada com os $50 \%$, na centrosema, pode ter afetado as possíveis reações do $\mathrm{P}$ disponibilizado com o cálcio advindo da calagem, pois a disponibilidade do $\mathrm{P}$ pode ter diminuído através da formação do fosfato tricálcico, cujo P precipitado não estaria disponível no período de tempo em que foi realizado o experimento.

Os valores obtidos com a porcentagem do $\mathrm{P}$ recuperado confirmam os resultados de Anghinoni \& Bohen (1974), em 40 solos do Estado do Rio Grande do Sul, e por Guss et al. (1990), em cinco solos do Estado de Minas Gerais, que verificaram um aumento linear na porcentagem do $\mathrm{P}$ recuperado em razão das doses de $\mathrm{P}$ aplicadas.

Os fosfatos naturais não alteraram significativamente o $\mathrm{pH}$ do solo, independentemente da dose de
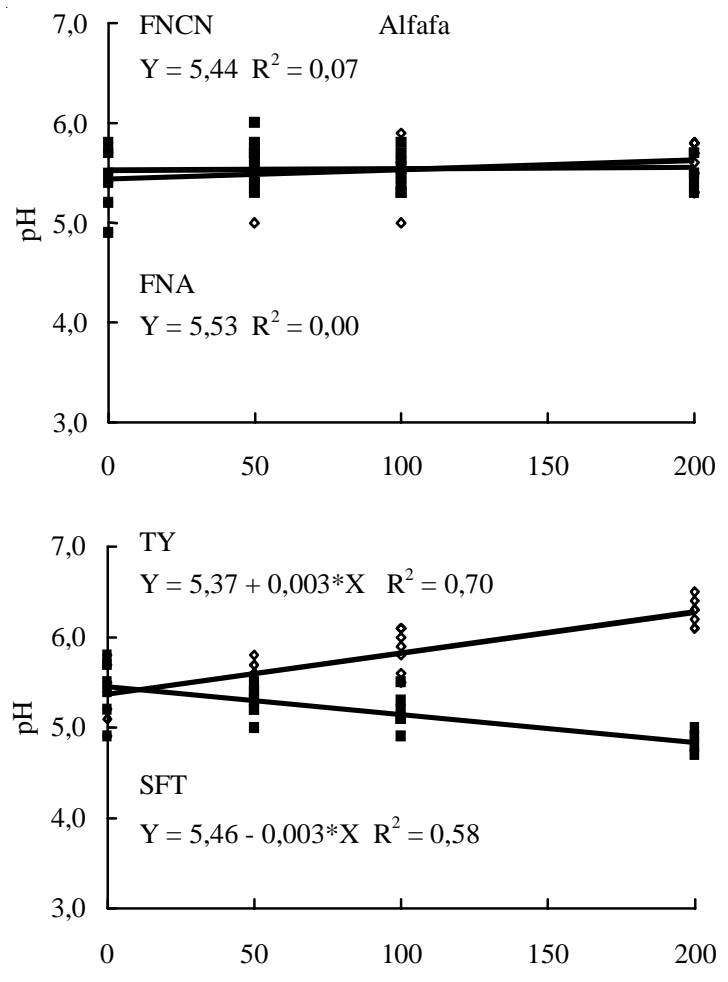

Fósforo $\left(\mathrm{mg} \mathrm{kg}^{-1}\right)$
$\mathrm{P}$ e da leguminosa (Figura 1). No caso do termofosfato Yoorin e do superfosfato triplo, houve uma alteração significativa do $\mathrm{pH}$, sendo que o primeiro aumentou e o segundo diminuiu o $\mathrm{pH}$ com o aumento das doses de fósforo.

A aplicação dos fosfatos naturais produziu pequena elevação, porém não significativa, do $\mathrm{pH}$ do solo na alfafa na ordem de 0,05 unidades quando a fonte de $\mathrm{P}$ foi $\mathrm{FNCN}$, e 0,01 unidades de $\mathrm{pH}$ por $50 \mathrm{mg} \mathrm{kg}^{-1}$ de P aplicado na forma de FNA (Figura 1). Quanto à centrosema, os incrementos nos valores de $\mathrm{pH}$ foram de 0,02 e 0,07 unidades para cada $50 \mathrm{mg} \mathrm{kg}^{-1}$ de $\mathrm{P}$, em relação ao FNCN e ao FNA, respectivamente.

As doses do termofosfato Yoorin produziram elevação significativa do $\mathrm{pH}$ do solo, na razão de 0,15 e 0,25 unidades de $\mathrm{pH}$ por $50 \mathrm{mg} \mathrm{kg}^{-1}$ de $\mathrm{P}$ aplicado na alfafa e centrosema, respectivamente (Figura 1). Resultados semelhantes foram obtidos por Braga \&
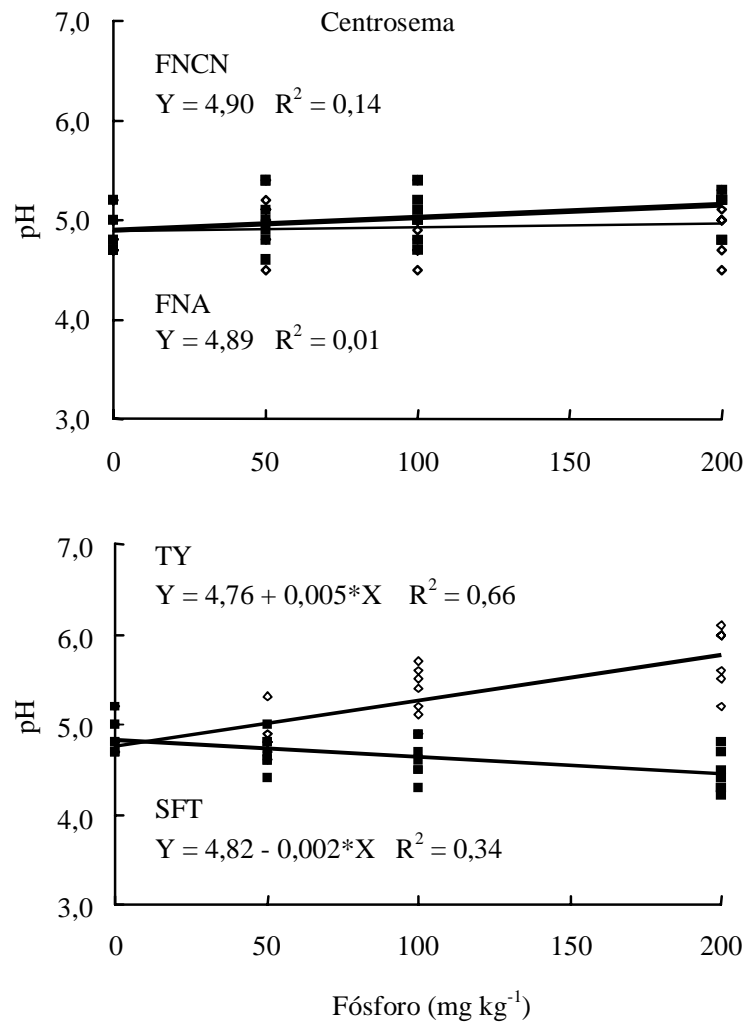

Figura 1. Efeito das fontes de $\mathrm{P}$, na média das seis amostragens, na variação do $\mathrm{pH}\left(\mathrm{em} \mathrm{CaCl}_{2} 0,01 \mathrm{~mol} \mathrm{~L}^{-1}\right)$ do solo cultivado com alfafa e centrosema. FNCN: fosfato natural da Carolina do Norte; FNA: fosfato natural de Arad; TY: termofosfato Yoorin; SFT: superfosfato triplo. *Significativo a 5\% de probabilidade pelo teste F. 
Amaral (1971) e Goedert \& Lobato (1984), ao verificarem que a aplicação de termofosfato aumentou significativamente o $\mathrm{pH}$ do solo. Assim, além da atividade do Si, que favorece a absorção de $\mathrm{P}$ (Souza \& Yasuda, 1995), a elevação do $\mathrm{pH}$ do solo, por causa do silicato, também possibilita um melhor aproveitamento do $\mathrm{P}$, e, conseqüentemente, maior produção de matéria seca.

Por outro lado, a aplicação do SFT reduziu significativamente o $\mathrm{pH}$ do solo, diminuindo 0,15 unidades de $\mathrm{pH}$ no cultivo de alfafa e 0,10 no de centrosema para cada $50 \mathrm{mg} \mathrm{kg}^{-1}$ de $\mathrm{P}$ aplicado no solo (Figura 1). Braga \& Amaral (1971) também observaram uma diminuição significativa do $\mathrm{pH}$ do solo, devido, provavelmente, à acidificação do solo causada pela hidrólise do SFT, dando origem à formação do ácido fosfórico. Apesar do superfosfato triplo ser considerado um fertilizante de reação neutra, outro fator que pode ter influenciado a diminuição do $\mathrm{pH}$ é o tempo de cura do fertilizante fosfatado; os fosfatos de fabricação mais recente podem apresentar um maior resíduo de ácido fosfórico ou sulfúrico do que os de fabricação mais antiga (Cekinski et al., 1990), causando o abaixamento do $\mathrm{pH}$ do solo.

\section{Conclusões}

1. A centrosema apresenta maior eficiência na utilização do $\mathrm{P}$ disponível do que a alfafa.

2. Com a sequiência de cortes, a produção de matéria seca obtida com os fosfatos naturais reativos tende a equiparar-se à das fontes mais solúveis.

3. O termofosfato Yoorin eleva o $\mathrm{pH}$ do solo, enquanto o superfosfato triplo o diminui e os fosfatos naturais apresentam reação neutra.

\section{Referências}

ALCARDE, J. C.; PONCHIO, C. O. A ação solubilizante das soluções de citrato de amônio e de ácido cítrico sobre fertilizantes fosfatados. Revista Brasileira de Ciência do Solo, Campinas, v. 3, p. 173-178, 1979.

ANGHINONI, I.; BOHEN, H. Avaliação da disponibilidade do fósforo para solos do Rio Grande do Sul. Agronomia Sulriograndense, Porto Alegre, v. 10, p. 127-136, 1974.

BRAGA, J. M.; AMARAL, F. A. Efeito de fontes de fósforo na variação de $\mathrm{pH}$ e disponibilidade de fósforo, cálcio e magnésio. Revista Ceres, Viçosa, MG, v. 17, p. 325$335,1971$.

CEKINSKI, E. Fertilizantes fosfatados. In: CEKINSKI, E.; CALMONOVICI, C. E.; BICHARA, J. M.; FABIANI, M.; GIULIETTI, M.; CASTRO, M. L. M. M.; SILVEIRA, P. B. M.; PRESSINOTTI, Q. S. H. C.; GUARDANI, R. (Ed.). Tecnologia de produção de fertilizantes. São Paulo: Instituto de Pesquisas Tecnológicas, 1990. p. 95-129.

CHIEN, S. H.; HAMMOND, L. L. A comparison of various laboratory methods for predicting the agronomic potential of phosphate rocks for direct application. Soil Science Society of America Journal, Madison, v. 42, p. 935-939, 1978.

COELHO, F. S.; VERLENGIA, F. Fertilidade do solo. Campinas: Instituto Campineiro de Ensino Agrícola, 1973. $384 \mathrm{p}$.

EMBRAPA. Centro Nacional de Pesquisa de Solos (Rio de Janeiro, RJ). Manual de métodos de análise de solo. Rio de Janeiro: Embrapa-CNPS, 1997. 212 p.

FASSBENDER, H. W.; BORNEMISZA, E. Química de suelos con énfasis en suelos de América Latina. San José: Instituto Interamericano de Cooperação para Agricultura, 1994. $420 \mathrm{p}$.

FENSTER, W. E.; LEÓN, L. A. Consideração sobre a fertilização fosfatada no estabelecimento e persistência de pastagens em solos ácidos e de baixa fertilidade na América Latina tropical. In: SÁNCHES, P. A.; TERGAS, L. E.; SERRÃO, E. A. S. (Ed.). Produção de pastagens em solos ácidos tropicais. Brasília: Centro Internacional de Agricultura Tropical, 1982. p. 127-141.

FÖHSE, D.; CLAASSEN, N.; JUNGK, A. Phosphorus efficiency of plants II: significance of root radius, root hairs and cation-anion balance for phosphorus influx in seven species. Plant and Soil, Dordrecht, v. 132, n. 1, p. 261-272, 1991.

GOEDERT, W. J.; LOBATO, E. Avaliação agronômica de fosfatos em solo de cerrado. Revista Brasileira de Ciência do Solo, Campinas, v. 8, p. 97-102, 1984.

GOEDERT, W. J.; REIN, T. A.; SOUSA, D. M. G. de. Eficiência agronômica de fosfatos naturais, fosfatos parcialmente acidulados e termofosfatos em solo de cerrado. Pesquisa Agropecuária Brasileira, Brasília, v. 25, n. 4, p. 521-530, abr. 1990.

GOEDERT, W. J.; SOUSA, D. M. G. de; LOBATO, E. Fósforo. In: GOEDERT, W. J. (Ed.). Solos dos cerrados: 
tecnologia e estratégias de manejo. São Paulo: Nobel, 1985. p. 129-163.

GUSS, A.; GOMIDE, J. A.; NOVAIS, R. F. Exigências de fósforo para estabelecimento de quatro leguminosas forrageiras em solos com distintas características físicoquímicas. Revista da Sociedade Brasileira de Zootecnia, Viçosa, MG, v. 19, p. 450-458, 1990.

LOBATO, E.; KORNELIUS, E.; SANZONOWICZ, C. Adubação fosfata em pastagens. In: MATTOS, H. B.; WERNER. J. C.; YAMADA, I.; MALAVOLTA, E. (Ed.). Calagem e adubação de pastagens. Piracicaba: Potafos, 1986. p. 145-174.

MALAVOLTA, E. Elementos de nutrição mineral de plantas. São Paulo: Agronômica Ceres, 1980. 251 p.

MEHLICH, A. Mehlich 3 soil test extractant: a modification of Mehlich 2 extractant. Communications in Soil Science and Plant Analysis, New York, v. 15, p. 1409-1416, 1984.

MEHLICH, A. New extractant for soil test evaluation of phosphorus, potassium, magnesium, calcium, sodium, manganese and zinc. Communications in Soil Science and Plant Analysis, New York, v. 9, p. 477-492, 1978.

MUZZILI, O. Adubação fosfatada no Estado do Paraná. In: OLIVEIRA, A.; LOURENÇO, S.; GOEDERT, W. J. (Ed.). Adubação fosfatada no Brasil. Brasília: EmbrapaDID, 1982. p. 61-101.
NOVAIS, R. F.; SMYTH, T. J. Fósforo em solo e planta em condições tropicais. Viçosa, MG: UFV, 1999. 399 p.

PIMENTEL-GOMES, F. Curso de estatística experimental. Piracicaba: Nobel, 1990. 468 p.

RAIJ, B. van. Seleção de métodos de laboratório para avaliar a disponibilidade de fósforo em solos. Revista Brasileira de Ciência do Solo, Campinas, v. 2, p. 1-9, 1978.

RAIJ, B. van; QUAGGIO, J. A.; SILVA, N. M. Extraction of phosphorus, potassium, calcium, and magnesium from soils by an ion-exchange resin procedure. Communications in Soil Science and Plant Analysis, New York, v. 17, p. 547-566, 1986.

SHELTON, J. E.; COLEMAN, N. T. Inorganic phosphorus fractions and their relationships to residual value of large applications of phosphorus on high phosphorus fixing soils. Soil Science Society of America Proceedings, Madison, v. 32, p. 91-94, 1968.

SOUZA, E. C. A.; YASUDA, M. Uso agronômico do termofosfato no Brasil. São Paulo: Fertilizantes Mitsui, 1995. $60 \mathrm{p}$.

TISDALE, S. L.; NELSON, W. L.; BEATON, J. D.; HAVLIN, J. L. Soil and fertilizer phosphorus. In: TISDALE, S. L.; NELSON, W. L.; BEATON, J. D.; HAVLIN, J. L. (Ed.). Soil fertility and fertilizers. New York: Macmmillan, 1993. p. 176-229. 